\title{
Threat assessment, sense making, and critical decision-making in police, military, ambulance, and fire services
}

\author{
Greg Penney ${ }^{1,6}$ (i) $\cdot$ David Launder $^{2} \cdot$ Joe Cuthbertson $^{3}$ (i) $\cdot$ Matthew B. Thompson ${ }^{4,5}$ (D)
}

Received: 2 September 2021 / Accepted: 6 January 2022 / Published online: 4 February 2022

(c) Crown 2022

\begin{abstract}
Military and emergency response remain inherently dangerous occupations that require the ability to accurately assess threats and make critical decisions under significant time pressures. The cognitive processes associated with these abilities are complex and have been the subject of several significant, albeit service specific studies. Here, we present an attempt at finding the commonalities in threat assessment, sense making, and critical decision-making for emergency response across police, military, ambulance, and fire services. Relevant research is identified and critically appraised through a systematic literature review of English-language studies published from January 2000 through July 2020 on threat assessment and critical decision-making theory in dynamic emergency service and military environments. A total of 10,084 titles and abstracts were reviewed, with 94 identified as suitable for inclusion in the study. We then present our findings focused on six lines of enquiry: Bibliometrics, Language, Situation Awareness, Critical Decision Making, Actions, and Evaluation. We then thematically analyse these findings to reveal the commonalities between the four services. Despite existing single or dual service studies in the field, this research is significant in that it is the first examine decision making and threat assessment theory across all four contexts of military, police, fire and ambulance services, but it is also the first to assess the state of knowledge and explore the extent that commonality exists and models or practices can be applied across each discipline. The results demonstrate all military and emergency services personnel apply both intuitive and formal decision-making processes, depending on multiple situational and individual factors. Institutional restriction of decision-making to a single process at the expense of the consideration of others, or the inappropriate training and application of otherwise appropriate decision-making processes in certain circumstances is likely to increase the potential for adverse outcomes, or at the very least restrict peak performance being achieved. The applications of the findings of the study not only extend to facilitating improved practice in each of the individual services examined, but provide a basis to assist future research, and contribute to the literature exploring threat assessment and decision making in dynamic contexts.
\end{abstract}

Keywords Situational awareness $\cdot$ Decision making $\cdot$ Firefighter $\cdot$ Police $\cdot$ Ambulance $\cdot$ Military $\cdot$ Recognition primed Heuristics · Bias · Threat assessment

Greg Penney

greg.penney@dfes.wa.gov.au

1 Department of Fire and Emergency Services, Perth, Australia

2 South Australian Metropolitan Fire Service, Adelaide, Australia

3 St John Ambulance Western Australia, Perth, Australia

4 School of Psychology, Murdoch University, Perth, Australia

5 Biosecurity and One Health Research Centre, Harry Butler Institute, Murdoch University, Perth, Australia

6 Edith Cowan University, Perth, Australia

\section{Introduction}

Effective emergency response to natural events, such as earthquakes, and deliberate events, such as terrorist attacks, involves the anticipation of emergency events and the actions taken during and immediately after to ensure that its affects are minimized (AIDR 2013). Within police, military, ambulance and fire service contexts this requires that responding personnel can (1) accurately make sense of dynamic and unfamiliar environments, (2) assess potential opportunities and threats to develop response plans, and (3) make critical decisions under significant time pressures (Cohen-Hatton et al. 2015; Perona et al. 2019; Penney 2019; Reay et al. 
2018). When confronted with a structure fire emergency, for example, a fire officer must process large amounts of visual and auditory information within short timeframes, including fire behavior, the location of casualties, available resources, and the construction of the structure involved. Simultaneously, they must be formalizing plans and contingencies to maximize the opportunity to rescue casualties, and to contain and extinguish the fire whilst also managing operations within the incident area and minimizing risk to responding firefighters. The cognitive processes associated with these abilities are complex (Reay et al. 2018; Stanton et al. 2009), susceptible to bias (Kahneman 2011; Seiler et al. 2010) and difficult to master (Perona et al. 2019).

Where incidents increase in scale and complexity, impacting large populations and geographic areas, response can last weeks or even months. Incidents now regularly cross regional, state, and national borders, resulting in a greater need to share information across agencies and for coordinated inter-agency response (Wilkinson et al. 2021). This subsequently poses additional challenges for decisionmakers (Alison and Crego 2008; Comfort 2007; Flin 1996; House et al. 2013; Pollock 2013; Coskun and Ozceylan 2011). Front line responders and incident managers alike must cooperate with different people in different organizations. In the UK, representatives from several organizations form Strategic Coordinating Groups that include fire, police, ambulance, civil resource organizations, health agencies, and government (Wilkinson et al. 2021). In the US and Australia equivalent personnel and agencies form Incident Management Teams (AIDR 2013; NIFC 2021). However, the challenge of multiple agencies in working together effectively during large scale disasters is ongoing (Alison and Crego 2008; Wilkinson et al. 2021; Pollock 2013).

It is tempting to think that the cause of the problem is one of communication breakdown between different personnel from differing agencies, and so collective understanding of the situation and operational requirements being lost in translation. Rather, we suggest the cause is that each service member is viewing the situation quite differently, through the lens of their training and standard operating procedure. In part this arises from variance in theories of decision making and threat assessment (Coskun and Ozceylan 2011), which ultimately guides operational response. And, traditionally, these theories have been segregated by service stream. The human factors of firefighters, paramedics, police, and military personnel are considered in isolation despite the similar circumstances of their dynamic and high consequence environments.

We posit that these services share greater similarities in operation and theory than previously identified. We suggest that progress can be made to improve inter-agency coordination as well as performance within individual service contexts if commonalities between services can be discerned and leveraged. A common understanding between services could then be developed, and existing research could be translated from service area to another. In turn this could promote research and resulting theories around emergency management that are service general rather than service specific.

Here, we present an attempt at finding the commonalities in threat assessment, sense making, and critical decisionmaking for emergency response across police, military, ambulance, and fire services. We present a review of relevant research across these areas of research and across these emergency services. First, we describe our aims and objectives, follow by our method. We then present our findings focused on five lines of enquiry that are relevant to emergency management: Language, Situation Awareness, Critical Decision Making, Actions, and Evaluation (terms defined in "Sect.1.1" below). We then consolidate these findings to reveal the commonalities between the four services.

This study is significant in that not only is it is the first to bridge this gap and examine decision making and threat assessment theory across the multiple contexts of military, police, fire and ambulance services, but it is also the first to assess the state of knowledge and explore the extent that commonality exists and models or practices can be applied across disciplines. The applications of the findings of the study not only extend to facilitating improved practice in each of the individual services examined, but provide a basis to assist future research, and contribute to the literature exploring threat assessment and decision making in dynamic contexts.

\subsection{Aims}

The scope of this study is multi-disciplinary, covering dynamic environments within civilian emergency services of policing, prehospital ambulance care, firefighting, and military operations. We aim to distill research in threat assessment, sense making, and critical decision making in dynamic emergency service and military environments. We also aim to discern the commonalities between services. In a similar approach to House et al. (2013), the aim was deconstructed into six distinct lines of enquiry:

1. Bibliometrics, allowing the 'spread' of research to be categorized by research design, service type, country of origin, year of publication, and, publication area;

2. Language, the terminology, definitions and descriptions within the research;

3. Situational Awareness (SA), threat assessment and sense making;

4. Critical Decision Making (CDM), the process used to decide on a course of action; 
5. Actions, the tasks both attempted and completed to execute the decision; and

6. Evaluation, the reflection of the decision and subsequent actions to determine whether or not a "good decision" has been made.

Whilst the justification for bibliometrics is self-explanatory, an iterative approach (Morgan and Nica 2020) to selecting lines of enquiry 2 to 5 was applied. In this way, initial lines of inquiry were revised by the research team during the conceptual phase of the study and refined, with the final lines of inquiry designed to bring meaning and identity a recurrent trends and variant manifestations as described by DeSantis and Ugarriza (2000). As such, the lines of enquiry were designed to capture and unify the nature or basis of the various context specific studies into a meaningful whole (DeSantis and Ugarriza 2000).

\section{Method}

Adapting the approach applied by House et al. (2013), this systematic review adhered to the set of scientific methods described by Petticrew and Roberts (2006) to limit bias. The resulting review presents a narrative approach adapted from Perona et al. (2019), and Launder and Perry (2014). The process comprised of four distinct phases (Fig. 1): (1) systematic identification of relevant studies; (2) critical appraisal of studies; (3) bibliometrics and narrative synthesis of data; and, (4) thematic analysis of findings. To achieve this a systematic literature review was completed.

\subsection{Phase 1: systematic identification of relevant studies}

To retrieve a large proportion of varied studies the search strategy was designed to be 'highly sensitive', as opposed to a 'highly specific' study which identifies a smaller number of more specific studies (Petticrew and Roberts 2006). Literature meeting the following criteria was included in the analysis: published peer-reviewed studies; reports; consensus guidelines; academic center, or professional association protocols detailing SA \& CDM in civilian emergency service or military environments; statistical analysis; and postincident reviews, inquiries, and inquests after incidents, published by government and non-government organizations.

The search strategy included only terms relating to, or SA \& CDM in dynamic emergency service and military environments (Table 1) that were identified during the preliminary literature review when determining the feasibility of the full study. A secondary search of bibliographies identified further literature for inclusion. Completed in October 2020, the review included English-language papers published

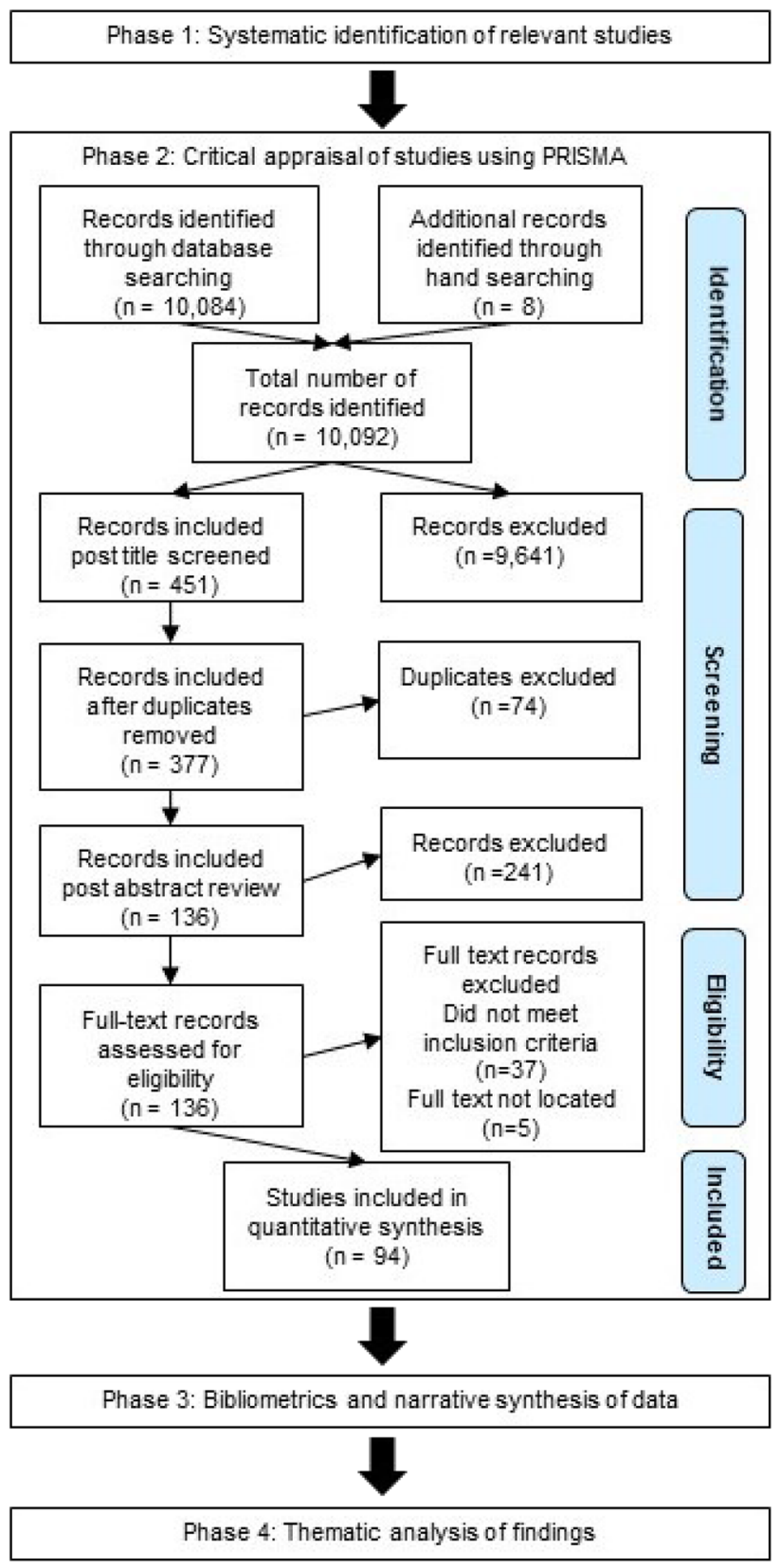

Fig. 1 Phases of the study

in the last twenty years (2001-2020) to ensure currency of evidence. Seminal papers from outside the date range were considered for inclusion where appropriate. Databases included Australian Federal Police Digest (Informit AFPD); ProQuest; Sage Journals Online; Science Direct; PubMed; and Cochrane Library. Non-English speaking literature, abstracts, citations, thesis, unverified or unsubstantiated opinion pieces, press or news media reports, nonpeer reviewed conference proceedings, articles that are not related to threat assessment and decision making in these environments were excluded. 
Table 1 Search terms used in the systematic literature review

Sources

Search terms

Limits
Informit AFPD, ProQuest, Sage Journals Online, Science Direct, PubMed and Cochrane Library

(Military OR Soldier OR Paramedic OR Ambulance OR Fire* OR Wildfire OR Bushfire OR Polic* OR Law Enforcement OR Command*) AND (Decision OR Risk OR Threat Assessment OR Dynamic)

English Language AND Published Between 2001 and 2020
Two review authors (GP \& JC) independently tested the search criteria and completed the initial search.

\subsection{Phase 2: critical appraisal of studies}

The initial search strategy yielded 10,084 studies for potential inclusion. Three authors (GP, JC \& DL) completed this process, with each stage initially completed independently by each author before group evaluation occurred. At each stage, a minimum of two of the three authors in the process had to agree for a study to progress to the next stage of review. Using this process, of the 10,084 records identified through the initial search strategy, 377 (excluding duplicates) had titles that were broadly identified as potentially relevant to the current study and progressed to a review of the abstract. The process was repeated with the abstracts reviewed against the eligibility criteria of the study, with 135 progressing to full manuscript review. Ninety-four (94) studies were ultimately included in the study. Results are presented according to the Preferred Reporting Items for Systematic Reviews and Meta-Analyses (PRISMA) checklist (Fig. 1).

Applying the approach described by House et al (2013, p. 3), a framework of assessment quality was established to critically appraise the 94 included studies. The framework appraised studies for their internal validity (the degree to which the research design, analysis and presentation have avoided biased comparisons), their external validity (the extent to which one can generalize findings to other settings) and the appropriateness of the data analysis and presentation. The findings of this analysis assisted prepare the reviews of each included study, which are provided as a supplementary file to this main manuscript.

The fourth author (MT) deliberately did not participate in the initial selection or synthesis of included studies, rather performing the role of 'red teaming', in other words he deliberately and critically attacked the study method and analyses in an effort to overcome cognitive errors including group think and confirmation bias..

\subsection{Phase 3: bibliometrics and narrative synthesis}

The narrative synthesis of findings was selected as it has proven useful for providing a comprehensive picture of the subject matter in question (McNeill and Chapman 2005) to guide new findings and conclusions (Fielding and Thomas 2001).

Data were initially quantitatively analysed, allowing the 'spread' of research to be categorized by research design, service type, country of origin, year of publication, and publication area. Research design was categorized as qualitative (did not yield numerical data/analysis), quantitative (yielded numerical data/analysis) or mixed method. Publication area was categorized as detailed in Table 2 .

Three authors (GP, DL, JC) completed the initial narrative synthesis in line with the five remaining lines of enquiry: Language, Situational Awareness (SA), Critical Decision Making (CDM), Actions, and Review. In an attempt to minimize bias that may develop by the authors working closely together on the study over a period of time, the fourth author (MT) was deliberately excluded from the initial synthesis and subsequently provided detailed critique and deliberate challenge once the initial review was complete. Any disagreements were resolved through discussion.

\subsection{Phase 4: thematic analysis}

Thematic analysis is a method for identifying, analysing and reporting patterns or themes within the data (Braun and Clarke 2006). An inductive thematic analysis, which seeks to identify broad trends arising from the literature itself (Braun and Clarke 2006), was applied to the results of lines of enquiry two to six, being: Language, Situational Awareness (SA), Critical Decision Making (CDM), Actions, and Review. In the context of the study, a centralized theme of commonality, or sharing of language, theories and processes was realized and explored. Applying the same approach as step three, three authors (GP, DL, JC) completed the initial thematic analysis, whilst the fourth author (MT) provided detailed critique and deliberate challenge once the initial analysis was complete. Any disagreements were resolved through discussion.

\section{Findings}

\subsection{Enquiriy line one: bibliometrics}

Database searches yielded a total of 10,084 articles, however, less than $1 \%(n=94)$ met the full inclusion criteria 
Table 2 Publication area categories and descriptions

\begin{tabular}{ll}
\hline Category & Description \\
\hline Emergency management & $\begin{array}{c}\text { The organization and management of the resources and responsibilities } \\
\text { for dealing with all humanitarian aspects of emergencies (prepared- } \\
\text { ness, response, mitigation, and recovery) } \\
\text { Medical and paramedical care in the prehospital environment }\end{array}$ \\
$\begin{array}{l}\text { Emergency medicine } \\
\text { Fire technology }\end{array}$ & Fire science (not a trade magazine) \\
Human factors & Ergonomics-human abilities and limitations \\
Medicine & Medicine in the hospital and clinic environment \\
Military & Military studies (not including military psychology) \\
Nursing & Nursing care and related studies \\
Policing & Police trade journals \\
Psychiatry & Psychiatry specific journals \\
Psychology & Psychology specific journals (including military psychology) \\
Risk & Risk assessment and management \\
Safety & Safety and occupational health hand safety \\
Not otherwise classified & Not otherwise classified \\
\hline
\end{tabular}

and were selected for the study (refer to the Supplementary Table S1). Seminal research, theories and definitions, for example, work by Endsley (1995), and Kahneman and Klein (2009), did not meet the specific search criteria but were cited by multiple included publications. They are, therefore, discussed as an additional text to this study but is not included as part of the systematic search results. A majority of all studies $(n=52)$ utilised a qualitative research methodology that did not result in the creation of numerical data, $17 \%(n=16)$ used a quantitative methodology, and $29 \%(n=27)$ used a mixed method approach. Of the qualitative studies 18 were literature reviews. A further four were systematic literature reviews having a mean 27.5 studies included for analysis. By service type, research related to ambulance paramedics accounted for $24 \%(n=23)$ of the studies, firefighting for $16 \%(n=15)$, military for $26 \%$ $(n=24)$, police for $33 \%(n=31)$, and blended research across services accounting for only $2 \%(n=2)$. None of the literature included in the study addressed more than two service types.

By publication area, Psychology accounted for the highest number of included studies at 29\% $(n=27)$, with Emergency Medicine (prehospital) accounting for the second highest at $19 \%(n=18)$, and both Medicine and Emergency Management accounting for the third highest at $5 \%$ ( $n=5$ each). The remaining studies were reasonably distributed throughout publication areas. By country of origin, the USA and UK tied for the most research at $23 \%(n=22)$, with collaborative research across countries accounting for $12 \%(n=11)$, and Australia accounting for the third highest at $11 \%(n=10)$. Analysis by country of origin and service identified the UK published the most studies in the ambulance $(n=9)$, Australia published the most firefighting studies $(n=5)$, the USA published the most studies related to policing $(n=14)$, and, the UK and USA equally published the most studies relating to military contexts $(n=5)$.

By year of publication, 2018 to 2020 collectively accounted for $39 \%(n=37)$ of all studies, with the remaining years resulting in a mean 3.3 studies published per year. Analysis by year of publication and service identified $78 \%$ $(n=18)$ of all ambulance paramedic related studies were completed between 2011 and 2020. By comparison, 80\% $(n=12)$ of all firefighting related studies, $58 \%(n=14)$ of all military related studies, and $84 \%(n=26)$ of all police related studies were published in that same date range.

Of the 94 publications, only four authors (Anderson, Cohen-Hatton, Jensen, and Shortland) published more than one study meeting the criteria. Whilst Shortland published three studies and examined both police and military contexts, the remaining authors published two studies each and within a single service context.

The results of the descriptive analysis suggest that research into threat assessment and decision-making has been historically 'siloed' from a service context perspective, without institutional consideration of how results and lessons learned may be applied across service contexts and jurisdictions. The recent popularity of research into the field (40\% of the studies being published since 2018), in addition to the limited number of authors with multiple publications, suggests that the study of threat assessment and decisionmaking, whilst not a new field, may be far from mature in regard to the identification of best practice decision making methods that are transferable across services and the varied contexts in which they must be applied. Our research suggests that there may be far more commonality regarding decision-making theory and processes between service contexts than is currently acknowledge within both the academia and the services themselves. 


\subsection{Enquiry line two: language}

Whilst absolutely consistency between service contexts (and even to some degree within service contexts) was not found, similar terminology, definitions and descriptions were present within the literature reviewed. In particular, fire services and the military regularly made reference to 'situational awareness' (Stanton et al. 2009; Launder and Perry 2014; Marusich et al. 2016; Catherwood et al. 2012; Godwin et al. 2015; Shortland et al. 2018), or synonyms including 'assessment' (Penney 2019; Martínez-Fiestas et al. 2020; Okoli et al. 2016), 'situation assessment' (Cohen-Hatton et al. 2015; Cohen-Hatton and Honey 2015), 'orientation' (Seiler et al. 2010; Bryant 2006) and 'perception' (Dodd et al. 2006; Martínez-Fiestas et al. 2020; Prati et al. 2013). In these texts, clear distinction was made between situational awareness, decision-making, plan formation, plan execution and dynamic review which ultimately formed part of the decision-making process. Alternative terminology included 'recognition' (Klein et al. 2010; Thomas 2020), whereby it was reported firefighters intuitively analysed dynamic information, and 'anomaly detection' (Curnin et al. 2020) whereby the study participant had to rely on critical analysis in the absence of identifiable cues or patterns. By comparison, within ambulance contexts the terms 'decision making', 'recognition', 'hypothetico-deductive reasoning' or 'fluid sense making' were applied (Ryan and Halliwell 2013). Both military and police studies referenced 'perceiving', 'threat assessment', 'high stress decision making' (Akinci and Sadler-Smith 2020; Bonner 2018; Gamble et al. 2018; Harris et al. 2017; Jederberg et al. 2002).

Consideration of 'Naturalist Decision Making', and 'Heuristics/Biases' approach (Launder and Perry 2014; Wilson et al. 2011; Akinci and Sadler-Smith 2020), 'experience', 'bias' and 'accountability' was present across all services (Cohen-Hatton et al. 2015; Perona et al. 2019; Launder and Perry 2014; Marusich et al. 2016; van den Heuvel et al. 2014; Gunnarsson and Warrén Stomberg 2009; Bakken and Gilljam 2003; Banks et al. 2020; Nja and Rake 2009; Verhage et al. 2018; Vickers and Lewinksi 2012). The theory of Recognition Primed Decision making was also cited repeatedly through the texts and across service streams (Cohen-Hatton et al. 2015; Cohen-Hatton and Honey 2015; Klein et al. 2010; Bonner 2018; Hine et al. 2019).

'Morality', 'ethics', and 'moral/ethical decision making' themes were present across all services (Penney 2019; Seiler et al. 2010; Harman et al. 2019; Ishoy 2016; Leibold et al. 2018; Murphy-Jones and Timmons 2016; Thompson et al. 2018), however, the full impact of these factors was not universally explored.

\subsection{Enquiry line three: situational awareness}

During this initial phase, the decision-maker makes sense of their surroundings by gathering and interpreting situational cues to establish what is currently on what is likely to happen. The most commonly applied term for this process across firefighting and military literature is situational awareness (Cohen-Hatton and Honey 2015; Launder and Perry 2014; Shortland et al. 2018). Noting the identified differences, the seminal definition of situational awareness (SA) by Endsley (1995, p. 36), being the "perception of the elements in the environment within a volume of time and space, the comprehension of their meaning, and the projection of their status in the near future", appears to define the process of information gathering, analysis and understanding across the dynamic environments of all service contexts (regardless of whether this process is considered intuitive or deliberate). The literature provides broad support that the development of situational awareness is a critical precursory phase of decision-making. For example, Cohen-Hatton et al. (2015), Perona et al. (2019), Penney (2019), Launder and Perry (2014), Marusich et al. (2016), Godwin et al. (2015).

Endsley (1995) defines three levels of situational awareness that remain applicable and relevant regardless of service or situational context. Level I situational awareness concerns knowing what information to look for and how to obtain that information. Level II situational awareness involves the comprehension and understanding of these situational cues to establish an understanding of the current situation. Finally, level III situational awareness refers to the ability to use the available information and understanding of what is happening to accurately predict future situations. Although different terminology is used, this requirement to identify and understand situational cues is consistently identified across the literature and service contexts.

There are specific differences in the nature of situational information that is required across police, paramedic, firefighting and military settings. For example, in high-risk police situations key situational information may include the incident type in risk level, the location (open or confined), and the number of persons involved and the level of public visibility. Consideration is also given to a potential suspect's build, gender, behavior and proximity. For example, Harman et al. (2019), Hine et al. (2019), and Verhage et al. (2018). In the literature describing paramedic decisionmaking, an analogous patient diagnostic process is described that highlights the required ability to critically analyse and rapidly form clinical impressions with minimal information. For example, Perona et al. (2019), Reay et al. (2018), and Murdoch (2019). In addition to identifying clinical symptoms, paramedics, like police officers must also consider the presence of bystanders and persons close to the patient being treated (Anderson et al. 2018). Furthermore, a distinction is 
made between experts and novice paramedics, with experts applying greater interconnectedness of conscious and subconscious processing to draw on information from multiple sources including professional learning and personal experiences (Perona et al. 2019). The same distinction is made between expert and novice military personnel in dynamic high threat situations (Godwin et al. 2015). Similarly, the literature concerning firefighting also supports this need to obtain key situational information that may include life risk, visible smoke and flames behavior, fuel load, occupancy, utilities, and the presence of hazardous materials (for example, Stanton et al. (2009), Launder and Perry (2014), Marusich et al. (2016), and Catherwood et al. (2012)).

There are commonalities in the information required across these first responder situations. For example, police, paramedics and firefighters frequently must gather situational information under intense time pressure (Penney 2019; Cohen-Hatton and Honey 2015; Launder and Perry 2014; Ågård et al. 2012; Anderson et al. 2018). In addition to the cues that are specific to each industry, these decisionmakers consistently seek information concerning the level of perceived risk, the availability of resources to manage the risk/threat and the presence of victims or civilians any information considered (for example, Penney (2019), Ågård et al. (2012), Anderson et al. (2019), and Hemmatjo et al. (2019)).

Next, as information is gathered it is used to establish a mental model of the situation or schemata (for example, Cohen-Hatton et al. (2015), Reay et al. (2018), and Kahneman and Klein (2009)). This mental model summarises the key situational information into a holistic picture that tells the decision-maker what is going on, including the level of risk/threat, the key factors that must be addressed, and potentially, how the situation will evolve (Endsley 1995). The ability to develop and maintain effective situational awareness may be impacted by a range of factors. These factors include both implicit and explicit biases (Harman et al. 2019), the volume of information and time available to process it (Penney 2019), cognitive limitations (Marusich et al. 2016), and the extent to which a situation is threatening to themselves or their colleagues. For example, sensory processes in police officers are affected in personally threatening situations have reported 'tunnel-vision' when attention becomes focused on potential weapons and other potentially critical situational information may be missed (Akinola and Mendes 2012; Harman et al. 2019; Verhage et al. 2018).

Differences in the way novices and experts establish and maintain situational awareness is also identified in the literature. For example, novices (and specifically inexperienced paramedics) demonstrate reliance on rule-based aide-memoires or checklists to ensure they consider critical factors and patient symptoms (Perona et al. 2019; Anderson et al. 2018). Therefore, novices must be informed, taught or advised what constitutes critical situational information (in their context), and what it means (Kahneman and Klein 2009; Ågård et al. 2012; Harman et al. 2019).

In contrast, experts have developed the ability to filter (or batch) critical situational information thereby reducing the total amount of information requiring mental consideration (Okoli et al. 2016). Experts employ interconnected conscious and subconscious processes that draw information from multiple sources that include professional training and personal experience (Perona et al. 2019). In these circumstances the process of both identifying situational cues and establishing awareness of their meaning may be intuitive and nearly instantaneous (Kahneman and Klein 2009; Bakken and Gilljam 2003). Finally, experts may progress nearly instantaneously from observing situational cues, establishing a mental model of what is happening and identifying a potential course of action (for example, Cohen-Hatton and Honey (2015), Kahneman and Klein (2009), and Launder and Perry (2014)). It is consequently reasonable to expect experts and novices may apply different strategies for establishing situational awareness that shape how they subsequently make decisions.

\subsection{Enquiry line four: critical decision making}

The literature identifies that personnel employ a range (or spectrum) of decision-making strategies, each defined by distinct characteristics (Seiler et al. 2010; Launder and Perry 2014; Bakken and Gilljam 2003).

The fastest decision strategies, including recognition primed, intuitive, value-based, or heuristics-based decisions are frequently classified as naturalistic (alternately Type 1 or System 1) strategies (Kahneman 2011; Klein et al. 2010; Kahneman and Klein 2009). These decisions may appear to seamlessly and instantaneously follow the development of situational awareness. Experts can apply these rapid decision-making strategies by immediately identifying information they have learned is critical, rapidly developing a mental model (schema) and recalling a satisfactory decision or solution from prior experience (Klein et al. 2010; Godwin et al. 2015; Oosterwold et al. 2018). Furthermore, experts may be able to make decisions on the basis of level II situational awareness (Cohen-Hatton et al. 2015; Cohen-Hatton and Honey 2015). Although differing terminology may be applied, the literature describes the frequent use of these rapid decision strategies by police, firefighting, paramedic and military personnel in low-time, high-risk settings (for example, Cohen-Hatton et al. (2015), Bakken and Gilljam (2003), and Harman et al (2019)).

A key advantage of these naturalistic decision strategies is the speed by which an effective decision can be made. Indeed, it has been posited that the management of low-time critical incidents such as fire require not only that accurate decisions are made; it also requires that such decisions are 
made intuitively (Okoli et al. 2016). The majority of decisions made by first responding fire officers are naturalistic (Cohen-Hatton et al. 2015; Cohen-Hatton and Honey 2015), and more specifically recognition primed, including up to $80 \%$ of initial fire ground decisions (Klein et al. 2010). Similarly, naturalistic decision strategies are described in lowtime policing situations that include 'shoot or no shoot' incidents (Harman et al. 2019; Verhulst and Rutkowski 2018). Military literature also describes the extensive use of a range of naturalistic decision strategies applied in dynamic situations. Sometimes referred to as combat dynamic intuition (Bakken and Gilljam 2003). This describes the ability of military personnel to make nearly instantaneous decisions in uncertain dynamic environments with low cognitive control and low conscious awareness (Militello et al. 2015). The application of naturalistic decision strategies appears less well defined in paramedic emergency situations. Although the use of these strategies by highly experienced paramedics is supported, they are often described in conjunction with prescriptive processes and protocols such as the emergency ambulance triage system (Reay et al. 2018; Murdoch 2019).

Furthermore, there are limitations to these naturalistic decision strategies. First, their effective use is reliant on extensive prior experience in an area of expertise (Klein et al. 2010). They should not be applied by less experienced decision-makers who are unable to filter and interpret key situational information and recall a satisfactory solution from prior experience (Bang and Liwång 2016). Furthermore, concerns were identified that these strategies may be susceptible to misdiagnosis and decision errors, particularly where a rapidly evolving situation is chaotic with time and emotional pressure (Perona et al. 2019; Marusich et al. 2016; Murdoch 2019). Experienced personnel may make poor decisions through overconfidence in areas outside their own field of expertise (Kahneman and Klein 2009). Finally, naturalistic strategies may not be appropriate in new or novel situations where the decision-maker lacks an experiential base (Curnin et al. 2020).

Despite the reported limitations, a key finding from the literature is that the majority of initial decisions made in rapidly changing, high-risk and uncertain situations by personnel across the sectors are naturalistic in nature (CohenHatton and Honey 2015; Klein et al. 2010; Launder and Perry 2014; Bakken and Gilljam 2003; Banks et al. 2020; Harman et al. 2019; Murdoch 2019; Okoli et al. 2016).

In contrast, decision strategies that involve the consideration of multiple options are more time intensive. These strategies, referred to as formal/classical (alternately Type 2 or System 2) include decision ladders, step-based protocols and procedures, complex problem-solving, creative, analytical and rational decision-making. These formal decision strategies share common elements including extended situational analysis (for example, risk identification, assessment and evaluation) and the identification and comparison of multiple options or courses of action. Prior to the development of naturalistic decision theory these formal strategies were used extensively to describe and teach decision processes across firefighting, policing, paramedic and most prominently, military settings (Klein et al. 2010; Banks et al. 2020; Shortland et al. 2018, 2020a, b).

A finding from the literature is that formal decision strategies remain in widespread use across the police, fire, paramedic and military sectors (Penney 2019; Reay et al. 2018; Seiler et al. 2010; Ågård et al. 2012; Akinci and SadlerSmith 2020; Bakken and Gilljam 2003; Banks et al. 2020; Harman et al. 2019; Hine et al. 2019; Murdoch 2019; Okoli et al.2016). In particular, the use of formal analytical decision-making processes is supported in situations where there is sufficient time to consider multiple options, for example, strategic military decisions that are not made in the field (Seiler et al. 2010; Shortland et al. 2018, 2020a, b(a)), where the consequences of deviating from the described process or previously identified solution are extreme, for example, police shoot-no shoot situations and paramedic emergency triage situations (Ågård et al. 2012; Hine et al. 2019; Murdoch 2019). Despite their potential rigor, the practical shortcomings of formal decision strategies in lowtime environments have become more obvious (Okoli et al. 2016). In low-time environments decision-makers can be overwhelmed by the influx of situational information (Banks et al. 2020; Okoli et al. 2016). Furthermore, the need to follow a prescribed sequential process when there is limited time may cause considerable tension or anxiety (Reay et al. 2018).

A hard distinction between naturalistic decision and formal decision strategies may not accurately reflect a more complex relationship between intuition and analysis observed in practice (Akinci and Sadler-Smith 2020). In particular, police and paramedics described challenges and stress associated with balancing the application of naturalistic and formal decision strategies (Reay et al. 2018; Akinci and Sadler-Smith 2020; Verhage et al. 2018) during what have been termed 'fuzzy tasks' (Verhulst and Rutkowski 2018). Additionally, decision strategies based on overlearning of evidence-based knowledge such as procedures, guidelines/protocols, mnemonics and aide-memoirs may also occupy a grey area in the middle of the decision-making spectrum (Perona et al. 2019). Procedures may be applied by experts when performing recurrent or routine aspects of tasks (Kahneman 2011), or to provide a predetermined decision for less experienced personnel (Perona et al. 2019; Reay et al. 2018; Seiler et al. 2010).

In summary, personnel across the sectors establish situational awareness and then make decisions using a range of strategies from the potential spectrum. Although the majority decisions made by experts in low-time situations are 
naturalistic decisions may also be made using formal analytical strategies when time is available, there is a prescribed organisational solution to a high-risk problem or where the decision-maker is an experienced (Perona et al. 2019; Penney 2019; Curnin et al. 2020; Harris et al. 2017).

Whilst the literature reviewed provides significant discussion regarding decision-making theory and methods, and what can affect an individual's ability to make a decision, it does not directly address how to determine whether a decision is indeed good or bad. From the research the resultant outcome is paramount to whether a decision is considered 'good' after the fact, perhaps the strongest example of this is the multiple police shooter-simulation studies where perceived bias is heavily scrutinised. The question of whether outcome is indeed the most appropriate measure of the suitability of a decision remains unanswered. It is also important to consider that whilst an effective and correct decision regarding an outcome or action may be made, plan formation and plan execution remain critical components to achieving a satisfactory outcome. Indeed, the research suggests that in many high threat and dynamic military, police, fire and ambulance environments, even if a perfect decision is made and effectively executed, the outcome may remain unsatisfactory or even bad, albeit not as bad as it had the potential to be. For example, consider an unavoidable situation that necessitates a difficult decision. A suitable decision-making process is followed, commensurate with the expertise and experience of the decision-maker, and results in the death of two personnel. The potential outcome arising from any other decision, being the death of six personnel, is avoided. Without the worse outcome to compare the original result to, how would the decision be reviewed? The limited research to explore this area (Penney 2019) occurred in the firefighting context and suggests that suitably experienced and qualified peers would be equally as likely to identify a decision as correct as incorrect across a range of incident contexts.

\subsection{Enquiry line five: actions}

Analysis of the actions arising from the decision-making process was not addressed in the literature as the formation of situational awareness or the decision making process. One aspect that was identified, however, was that regardless of service, in the absence of translating decisions into timely and appropriate physical action, in other words 'Plan Execution' (Cohen-Hatton et al. 2015; Cohen-Hatton and Honey 2015; Shortland et al. 2018), even 'good' decisions become ineffective (Launder and Perry 2014; Bakken and Gilljam 2003; Harman et al. 2019).

Where the decision-maker was in a position of command authority and not personally able to complete the required physical tasks, for example an Incident Controller or ranking officer, clear and effective communication to those responsible for completing the physical tasks was considered an essential action (Launder and Perry 2014; Marusich et al. 2016; Bakken and Gilljam 2003; Verhulst and Rutkowski 2018). The level of trust subordinates had in decision-makers was also identified as a factor influencing whether the subordinate would execute the directed action or complete their own analysis of the situation and take a different course of action (Marusich et al. 2016).

Where the decision-maker was also responsible for completing actions, a number of findings were identified. In the absence of immediate personal threat and when faced with either high levels of uncertainty, situations not familiar to the responder, or where a fear of personally adverse outcomes are present, inexperienced personnel and those seeking confirmation of their decision theories are likely to be risk adverse, default to following standardised protocols and may ignore more dynamic and potentially beneficial processes (Reay et al. 2018; Ågård et al. 2012; Anderson et al. 2018; Bakken and Gilljam 2003; Burrell et al. 2013; Knighton 2004; Larsen 2001; O'Hara et al. 2015; Oosterwold et al. 2018; Vickers and Lewinski 2012). In the presence of perceived immediate personal physical threat, actions are more likely to be self-preservative even when further analysis may have resulted in either the perceived threat being dismissed, or more appropriate courses of action being identified (Correll et al. 2007; Gamble et al. 2018; Harman et al. 2019; Lima and de Araujo 2018; Nieuwenhuys et al. 2015). In such instances, there was some evidence to suggest there is little difference between inexperienced and experienced personnel in the time taken to complete the action, however, experienced personnel took less time to initiate that course of action compared to inexperienced personnel (Vickers and Lewinski 2012).

\subsection{Enquiry line six: evaluation}

The literature identifies two distinct forms of 'evaluation' behaviors that occur across the industry contexts examined. First, there are dynamic review processes that occur during an extended situation where multiple decisions are made (Launder and Perry 2014; Ågård et al. 2012; Bakken and Gilljam 2003). Next, there are review behaviors and subsequent actions that may be undertaken following the conclusion of a situation to determine whether 'good decisions' have been made.

Dynamic review occurs in the majority of incident settings where once one decision is made further decisions will usually be required subsequently (Banks et al. 2020). These processes involve the reconsideration of situational factors to identify whether, following the implementation of initial decisions, the situation has changed as predicted (for better or worse) (Launder and Perry 2014). Consistent with the previous themes, prior experience influences 
the effectiveness of this dynamic review across each of the disciplines reviewed (Cohen-Hatton et al. 2015; Perona et al. 2019; Launder and Perry 2014; Banks et al. 2020). First, experience allows decision-makers in complex, rapidly evolving situations to more rapidly identify deviations (or threats) from the predicted mental model and generate a larger number of possible causes and consequences to explain events due to their broader perspective than novices (Launder and Perry 2014; Bakken and Gilljam 2003). Next, highly experienced personnel are less likely to react emotionally or employ excessive force under stress than novices (Harman et al. 2019). Furthermore, if emerging situational cues imply intuitive decisions could be wrong, experienced decision-makers may shift to formal or system 2 strategies, replacing intuition with careful reasoning (Kahneman 2011; Kahneman and Klein 2009).

Next, consider the review actions that may occur following an emergency, police or military incident. These actions involve retrospective analysis and evaluation of events and decisions made. However, there is minimal evidence of structured review process in critical decision making in the reviewed literature that guides practitioners in evaluation of decisions either real time or post event. Of the services examined, literature concerning prehospital care identified a framework of decision review and care intervention based on changes in patient presentation due to treatment effect (or lack thereof). However, like many services this decision review was predominantly informed by experience and pattern recognition. Interestingly, Gunnarsson and Warrén Stomberg (2009) noted that nonverbal expressions and behaviors by team members resulted in decision making reflection, it would appear that decision reflection was driven by behavior rather than an unbiased evaluation to validate (on invalidate) decisions. The findings of Gunnarsson and Warrén Stomberg (2009) are congruent with Bakken and Gilljam (2003) who showed evidence of confirmation bias in decision-making and failure to critically analyse decisions where outcomes were accepted.

The literature supports a belief that post-incident review through reflection, debriefing, and coaching, supports learning and the collective development of intuitive expertise and decision-making (Akinci and Sadler-Smith 2020). Furthermore, reflection and feedback appear vital to the development and maintenance of expertise by facilitating the restructuring of knowledge as experiences and outcomes are appraised and measured against actions (Perona et al. 2019). Moreover, there are factors that influence the effectiveness of review actions in developing future decision-making. For example, shallow reflections on action and lack of knowledge of outcomes are likely to create or develop biased, prejudice, and inaccurate intuitions (Akinci and Sadler-Smith 2020). However, the development of highly prescriptive and inflexible post-incident review methodologies may provide no better alternative. For example, resuscitating decisionmaking research designs commonly involve retrospective analysis of cardiac registry data and clinical records. However, these methodologies may not capture the complex and idiosyncratic experience of resuscitating decision-makers or other operating in dynamic, high-risk situations (Anderson et al. 2019).

Effective experiential learning may only occur under certain conditions: it requires accurate and immediate feedback about the relation between situational conditions and the appropriate response (Kahneman 2011; Bakken and Gilljam 2003). The inability to identify common and consistently applied review strategies in the literature may be symptomatic of the broader lack of a common and consistent incident decision-making framework at this time. In the absence of such a framework paramedic, fire, police and military agencies are independently developing localised solutions with the effectiveness dependent on each agencies understanding and application of research and evidence-based practices.

\section{Discussion}

\subsection{Commonalities between service disciplines}

In regard to the question of commonality across the disciplines explored, in other words, "to what extent is there commonality between military and emergency services in regards to research and evidence-based threat assessment and decision making?", we propose that there is greater commonality than currently identified in the individual pieces of research reviewed. Commonality across services was found in language, theory, and processes. Although there are differences in the nature of the information considered across police, paramedic, firefighting and military settings there are nonetheless commonalities in the processes by which information is gathered, interpreted and applied as a precursor to decision-making. This commonality suggests Endsley's (1995) seminal definition of Situational Awareness, being the "perception of the elements in the environment within a volume of time and space, the comprehension of their meaning, and the projection of their status in the near future", not only remains current 26 years after its publication, but the most encompassing term and accurate definition for this initial phase of the decision-making process regardless of context. Situational Awareness provides a transferable terminology and definition that can be applied across multiple industry contexts more effectively than more narrow and specific terms such as diagnosis, threat assessment or size-up. Additionally, situational awareness provides a conceptual framework describing a common process of identifying and interpreting contextually specific information 
and projecting potential outcomes. Within this framework, knowing what information to seek, how to find it and what it means becomes critical.

The term situational awareness better describes the broader and more holistic cues and information sets identified in the literature and considered by decision-makers than more narrow terms such as 'threat assessment'. For example, a soldier, paramedic, police officer, and firefighter will regularly find themselves in danger, however, to make sense of a threat without greater appreciation of the true context of the situation (regardless of whether it is an explosive device, combative brain-injured patient, unstable structure, or armed offender) is to ignore the relevant political, moral, legal, organisational, cultural, and other factors that may influence whether the decision is acceptable, suitable or correct.

Furthermore, situational awareness describes processes that are applicable where there is time to identify, interpret and analyse information as well as low-time, high-risk situations. Specifically, the term encompasses both formulaic, deliberate and analytical processes that may be taught explicitly to novices and intuitive nearly instantaneous processes applied by experts.

Therefore, although differences will remain in the nature of information considered by the services examined in this research, we find there are significant and important commonalities concerning the behaviors employed to establish 'situational awareness' and that there is consensus that this awareness is the precursor to effective decision-making. Significantly, this identified commonality has implications for developing greater consistency between the fire, police, military and paramedic sectors included in this research both operationally and in the training and development of personnel. The results of the study suggest that by embedding knowledge of the process of looking for, and assessing all the elements of Situational Awareness, and recognising that all dynamic situations are governed by 'rules of engagement', both decisions and outcomes may be improved across service contexts. Further, the extent of research supporting Situational Awareness as a foundation of decision making in part addresses the questions regarding scientific validity of the concept of situational awareness raised by Carsten and Vanderhaegen (2015), as it is indeed a concept that is not only common across services but has a wealth of deep scientific support across decision-making models and theories, even when those theories that do not necessarily agree on other aspects.

\subsection{Commonality in theory}

The researched literature identifies commonality in the description of decision-making processes employed across these industries. Although industry specific terms are applied, these terms repeatedly identify two common and major decision-making methodologies. First, the literature describes naturalistic (or intuitive/type 1) strategies that are employed in the majority during low-time situations. These intuitive strategies may be further delineated to include recognition-primed, value-based, and 'overlearned procedural' decisions. The naturalistic strategies involve a nearly seamless transition from the development of situational awareness to a decision. However, the effectiveness of these decisions is dependent on the experience, knowledge and confidence of the decision-maker and the relevance, clarity and simplicity of organisational procedure and preparatory training.

The literature identifies the widespread use of 'formal' (or analytical/type 2) decision strategies across the police, fire, paramedic, and military sectors. These formal strategies involve the consideration of multiple options and, therefore, are primarily employed in situations where an immediate decision is not required. Another commonality across service streams linked to formal decision-making is the dependence of the success of the decision-making process, is the setting of goals or objectives. Contrary to a small minority of the studies reviewed, the greater majority of the research suggests that almost all personnel have either inherent or deliberate goals they are trying to achieve. These goals may be automatic or subconscious, for example staying alive during a shoot-out with an armed offender; determined by organisational doctrine, for example the protection of life, property and the environment; or deliberately determined by an individual, for example where conflicts between goals, values or ethics occur. The research supports the notion that depending on the experience and personal attributes of the individual, the speed and success of the formation of the plan will vary.

During initial learning, novice military and emergency services personnel require assistance and supporting physical stimuli (otherwise known as perceptual clues) to enable neural pathways to be developed and 'sense' to be made from the overwhelming data and sources of information that they are exposed to (Kahneman 2011; Kahneman and Klein 2009; Launder and Perry 2014). As neural pathways and pattern matching occur over time, the students can make sense of increasingly complex information within reduced timeframes (Kahneman 2011), allowing them to effectively assess and manage risk (Penney 2019). As their decisionmaking abilities develop, experienced firefighters, incident managers, and emergency responders are able to progressively extend and adapt decision making to situations with greater speed than previously able (Kahneman and Klein 2009). With experience and mastery of their profession, emergency responders are able to recognise patterns from prior incidents, subconsciously plan and even pre-emptively respond to changes in incident dynamics (Cohen-Hatton and Honey 2015; Klein et al. 2010). Although a hard delineation between naturalistic and formal decision-strategies may not 
reflect the manner in which decision-makers select from a 'spectrum' of decision strategies, the terminologies nonetheless provide a practicable and common nomenclature that may be applied and communicated across these industries.

Next, the literature established that the effectiveness of decisions is dependent on the effectiveness of their translation into action. Areas of commonality across fire, police, military and paramedic settings included the individual capabilities and experience of the decision-maker, or where this individual was reliant on the actions of others, the effectiveness of communication, coordination and control and credibility and confidence of personnel involved. This identified commonality of 'action' behaviors supports the ongoing development of systems such as the Australasian Interagency Incident Management Systems (AIIMS) that seek to foster consistency of incident management practice and behavior across multiple responding agencies.

Finally, the literature identifies commonality concerning the review of decisions in fire, police, military and paramedic settings. Across these industries both dynamic review, that is review of the evolving situation, decisions made and their execution during an incident, as well as more formal and considered review that occurs following the conclusion of an incident. Whereas the effectiveness of dynamic review appears to be strongly linked to the experience of the decision-maker (irrespective of industry) the effectiveness of post-incident review is dependent on the presence of a consistent framework for both making and evaluating decisions.

This research supports the notion that an individual's experience and personal attributes strongly influences their ability to make sense of a situation and respond appropriately. It is almost impossible for inexperienced personnel to successfully utilise Recognition Primed Decision (RPD) making, regardless of service context. In the same manner, even highly experienced and capable personnel will be unable to exercise RPD in unfamiliar unique experiences. Further, the research suggests that there is a unique threshold, dependent on both the individual and the situation encountered, for which RPD will be able to be successfully implemented to achieve an acceptable outcome. Inexperienced personnel will almost always use a deliberate analytical decision process, which may be enhanced by organisational guidelines or protocols aligned to a certain outcome or goal. As an individual gains greater knowledge, and becomes more experienced and skillful, their ability to make sense of a situation, formulate a plan, and execute it increases. With true expertise, the speed at which this process occurs may make it appear that the individual is pre-emptively responding even before a threat is apparent. In this way, the research suggests that as opposed to the decision-making process being naturalistic, heuristic or analytical, these processes are actually part of a single decision-making spectrum. We argue the process applied in a specific situation depends as much on the decision maker's individual characteristics (training, bias, utility, expertise, prior experience, intelligence, self-awareness, health, emotion etc.) as it does on the context of the individual situation (time available, level of self-threat, consequences etc.).

\subsection{Commonality in processes}

Variance in the accepted process by which decisions are made between services was identified. Popular amongst fire services and based on seminal research by Klein et al. in 1995, published in (Klein et al. 2010), the theory of Recognition Primed Decision Making (RPDM), provided a model to describe the process used by firefighters to make critical decisions during complex and dynamic operations. Recently, however, alternate theories (SAI Global 2013; Burrell et al. 2013), and variations (Cohen-Hatton et al. 2015; CohenHatton and Honey 2015) to RPDM have been proposed. Within military contexts, various command decision making frameworks exist including the Interactional Dual-Process model of moral decision making (Seiler et al. 2010); Observe-Orient-Decide-Act Loop and Critique-ExploreCompare-Adapt Loop (Bryant 2006). Cognitive processes and threat assessment within ambulance and police contexts are typically identified as fluid sense making and experience or bias centered as opposed to the defined processes of other services (Perona et al. 2019; Marusich et al. 2016; van den Heuvel et al. 2014; Gunnarsson and Warrén Stomberg 2009). Whilst the contribution of service specific research to the military, police, fire and ambulance contexts cannot be denied, there is an absence of cross-disciplinary research in the field.

However, our research identifies common threat assessment and decision-making elements that can potentially be used to develop a framework or spectrum of decision-making across these dynamic settings. These common elements include the development of situational awareness (Endsley 1995), the application of decision strategies from a potential spectrum that includes both rapid, naturalistic (intuitive) and formal (analytical) decisions, as well as behaviors required to enact and review decisions (both dynamically and formally). One member of the research team has previously published a decision framework consistent with this approach within the specific context of rapidly evolving urban firefighting (Launder and Perry 2014). However, this framework did not consider or seek to address commonality across paramedic, police or military settings. However, this research provides the opportunity to consider the broader extension of a consistent and common model that includes the identified decision-making elements across these industries.

Therefore, we suggest a framework or spectrum of decision-making which provides consideration of existing prominent and future relevant decision-making models, and 
which is transferable across the industries studied, can be developed. We suggest that by understanding the types of available decision-making processes and methodologies identified in the research and available on the decision making-spectrum, as opposed to confining operational decisionmaking to a single process or method, all of the processes identified in the texts reviewed can be readily adapted to suit situational contexts within each service. The research suggests that the processes employed to make decisions may only be half the issue. Much like learning styles, the most appropriate decision-making process for an individual will vary depending on the individual's unique characteristics or attributes including but not limited to experience, utility, intelligence, emotional intelligence, and education. The most appropriate decision-making process will also depend on the health of the individual, with fatigue, emotion and pain all having the potential to impact the decision arrived at in any given situation. The research suggests that whilst experience cannot be 'trained into' a person, one of the most effective ways to pre-emptively embed an effective decision-making process is regular high fidelity (potentially deconstructed) training environments that can introduce uncertainty, time restriction, and the perception of danger in a controlled manner. In the field, practices such as team decision-making (similar to that used by airline cockpit crews during emergencies), and distributed decision making so that an individual's decision-making utility is not exceeded, may also enhance the use of appropriate decision-making processes.

\subsection{Application}

The potential practical application of these findings within the various contexts extends to both the training and operational environments. Using the field of mathematics as an analogy, it is inappropriate to apply calculus in every situation such as simple addition just as it is inappropriate to expect a lower school student to complete advanced differential equations. In the same way, to develop a broad range of decision-making abilities for different situations, students and novice commanders within emergency and military services should be taught a range of decision making theory and models from which they can then select the most appropriate for the specific situation they find themselves in. At the same time, emergency and military services may need to consider whether they require students to have a minimum level of experience if they are going to teach experience reliant decision-making models such as RPDM and naturalist decision making, particularly for high consequence low frequency events that students and even experienced personnel may have limited exposure to. Where such models are intended to be taught in the absence of the required foundation experience, services may need to invest in scaffolded learning much in the same way that the basic principles of mathematics are established and built upon to enable students to progress from basic addition, to calculus, to differential equations and so on.

Within the operational environment the findings of this research can be applied through the potential adoption of frameworks such as the Cynefin framework (Snowden 2002) or SPAR framework (AFAC 2020) which promote adaptable and different sense-making and decision-making approaches, such as those models discussed in this study, depending on the complexity and novelty of the situation encountered. The findings can also be applied through deliberate efforts to increase commonality between services through joint learning and exercises involving decision-making personnel from different services who will be required to work effectively together to successfully coordinate the safe resolution of high consequence low frequency and time critical events.

From an autonomous systems perspective such as those proposed by Vanderhaegen (2021), the findings of this research and proposed applications may be perceived as a double-edged sword. Whilst increased commonality between human systems involving multiple decision-makers may assist the development of automated systems within the decision-making domain, increasing the range of processes relied upon by human decision-makers may potentially complicate attempts to automate such processes. It is suggested further research is required in this specific field to fully appreciate any impacts.

\subsection{Limitations}

In excess of 10,000 separate research artifacts were initially captured across six academic databases in this systematic literature review and 94 studies were ultimately included for full analysis, being 3.4 times greater than the average number of studies reviewed in other systematic literature reviews completed in the field to date. Despite this, it is acknowledged that research in other languages and non-peer reviewed studies may provide additional and valuable information. New research in the field is also constantly being published so it is important to acknowledge this study is completed at a point of time.

The search strategy for the study was specifically designed to be 'highly sensitive' and retrieve a large proportion of varied studies across the various emergency service and military contexts, as opposed to a 'highly specific' study which identifies a smaller number of more specific studies which would result in reduced studies being reviewed. Following several reviewers' comments regarding the original search not being broad enough, the terms were increased to include "law enforcement", "wildfire" and "bushfire". Whilst these yielded an additional 391 initial results (four being duplications of already included studies), no additional studies were ultimately included for full review as they did not meet the 
required inclusion criteria. This provides a level of confidence that whilst the search may not have been exhaustive, it certainly met the criteria for a plateau of diminished returns and the final set of included studies remains a reasonable basis for drawing conclusions. However, as we discussed in our findings regarding the commonality of language between services, absolute commonality was not present. This leaves open the possibility that some relevant yet uncommon terms, and, therefore, relevant studies, may have been missed.

Acknowledging the potential for bias in the selection of studies for inclusion in the research, the study deliberately required three authors to independently assess potential studies for suitability with any disputes addressed by consensus decision. The fourth author deliberately did not participate in the initial selection or synthesis of included studies, effectively performing the role of 'red teaming', i.e. in other words he deliberately and critically attacked the study method and analyses in an effort to overcome cognitive errors including group think and confirmation bias.. Despite this, it is acknowledged that the research team are all Australians (albeit from different service and research backgrounds) and may be biased by their shared cultures.

\section{Conclusions}

To better understand the potential for improvement within individual services, and during inter-agency emergency and disaster response, we attempted to discern commonalities across threat assessment, sense making, and decision making in dynamic emergency contexts between military, police, ambulance, and fire services. We systematically identified and critically appraised relevant studies, before completing a narrative synthesis and thematic analysis of those relevant to the research theme.

Whilst absolutely consistency in language between service contexts (and even to some degree within service contexts) was not found, similar terminology, definitions and descriptions were present within the literature reviewed, in particular fire services and the military. Despite the differences in operating environment and the nature of situational information required between services, commonality was found in the way novices in the different services make sense of their environments compared to more experienced personnel within the same service. The application of mental models, both intuitive and deliberate, to process this information and make critical decisions was also common across services, even if the models themselves differed. Common across all services and contexts was the belief that in the absence of translating decisions into timely and appropriate physical action, even 'good' decisions become ineffective. To execute the necessary actions to support good decisions, the need for clear communication and trust between decision-makers and those executing actions was consistently identified across all services.

We suggest that institutional restriction of decision-making to a single process at the expense of the consideration of others (for example institutional requirement to use RPDM as opposed to considering System 1 and 2), or the inappropriate training and application of otherwise appropriate decision-making processes in certain circumstances may increase the potential for adverse outcomes, or at the very least restrict peak performance being achieved. For example, attempting to apply RPDM where the user has insufficient experience with which to appropriately recognize the situation and recall an appropriate response, or attempting to apply a deliberate analytical approach where an instant decision is required. Whilst the specific contexts and circumstances of incidents between military, police, fire and paramedic operations and incidents will inevitably differ, ignoring the potential application of research across service streams may also serve to limit improvements in performance. To improve threat assessment and decision-making in military and emergency services contexts, and to address the uncertainties identified in this study, further research is recommended to define what differentiates a good and bad decision, and how this determination can be made.

The apparent lack of a framework or spectrum of decision-making common across service contexts in itself limits the potential to evaluate decisions (and their outcomes) across distinct industry settings. For example, although firefighters, police officers and paramedics may respond to a single multi-agency emergency incident they will at present apply differing terminology, procedures, controls structures and review processes. Without a common framework or spectrum to work within, or at least increased commonality between services, we posit that the inconsistency in conclusions as to whether a decision was good or bad between personnel within the same service context as previously reported in Penney (2019), let alone different services, will continue unaddressed. The development of a common yet scalable framework or spectrum of decision-making which provides consideration of existing prominent models such as those reviewed in this paper, as well as allowing for the incorporation of future relevant decision-making models, will have the additional benefit of providing a consistent evaluative framework that allows the evaluation of both the decision-making processes and behaviors applied, and their ultimate outcome.

Supplementary Information The online version contains supplementary material available at https://doi.org/10.1007/s10111-022-00694-3.

Acknowledgements Laura Toose, Alecia Wood and Clare Wallwork are thanked for their proofing and formatting assistance. 
Author contributions GP, DL, JC: conceptualization; GP, DL, JC, MT: methodology; GP, JC: validation; GP, DL JC, MT: formal analysis; GP, DL, JC: investigation; GP: data curation; GP, DL, JC: writing - original draft preparation; GP, DL, JC, MT: writing - review and editing; GP: project administration. All authors have read and agreed to the published version of the manuscript.

Funding Open Access funding enabled and organized by CAUL and its Member Institutions. This research received no external funding.

Availability of data and material The summary of all studies included in the final review are provided as a supplementary file.

\section{Declarations}

Conflict of interest Two authors (G.P. \& D.L.) work for state government fire and emergency services. J.C. works for a not-for-profit ambulance service. All statements expressed in this article are those of the authors and do not necessarily reflect the official opinion or policies of any of their affiliated institutions, civilian, military, government, or other. Two of the authors (G.P. and D.L.) have previously published in this field and each have a single research paper included in the study.

Open Access This article is licensed under a Creative Commons Attribution 4.0 International License, which permits use, sharing, adaptation, distribution and reproduction in any medium or format, as long as you give appropriate credit to the original author(s) and the source, provide a link to the Creative Commons licence, and indicate if changes were made. The images or other third party material in this article are included in the article's Creative Commons licence, unless indicated otherwise in a credit line to the material. If material is not included in the article's Creative Commons licence and your intended use is not permitted by statutory regulation or exceeds the permitted use, you will need to obtain permission directly from the copyright holder. To view a copy of this licence, visit http://creativecommons.org/licenses/by/4.0/.

\section{References}

AFAC (2020) Decision Making Under Presssure - A resource for Incident Management Teams. Australasian Fire and Emergency Authorities Council, Victoria, Australia

Ågård A, Herlitz J, Castrén M, Jonsson L, Sandman L (2012) Guidance for ambulance personnel on decisions and situations related to out-of-hospital CPR. Resuscitation 83(1):27-31. https://doi.org/10.1016/j.resuscitation.2011.07.028

AIDR (2013) Australian institute of disaster resilience glossary. https://knowledge.aidr.org.au/glossary/?wordOfTheDayId= $\&$ keywords $=\&$ alpha $=\mathrm{R} \&$ page $=2 \&$ results $=50 \&$ order $=\mathrm{AZ}$. Accessed June 2021

Akinci C, Sadler-Smith E (2020) "If something doesn't look right, go find out why": how intuitive decision making is accomplished in police first-response. Eur J Work Organ Psy 29(1):78-92. https://doi.org/10.1080/1359432X.2019.1681402

Akinola M, Mendes WB (2012) Stress-induced cortisol facilitates threat-related decision making among police officers. Behav Neurosci 126(1):167. https://doi.org/10.1037/a0026657

Alison L, Crego J (2008) Policing major incidents: leadership and major incident management. Willan Publishing

Anderson NE, Gott M, Slark J (2018) Beyond prognostication: ambulance personnel's lived experiences of cardiac arrest decision-making. Emerg Med J 35(4):208. https://doi.org/10. 1136/emermed-2017-206743

Anderson NE, Slark J, Faasse K, Gott M (2019) Paramedic student confidence, concerns, learning and experience with resuscitation decision-making and patient death: a pilot survey. Australian Emerg Care 22(3):156-161. https://doi.org/10.1016/j.auec. 2019.07.001

Bakken BT, Gilljam M (2003) Dynamic intuition in military command and control: why it is important, and how it should be developed. Cogn Technol Work 5(3):197-205. https://doi.org/ 10.1007/s10111-003-0123-1

Bang M, Liwång H (2016) Influences on threat assessment in a military context. Def Secur Anal 32(3):264-277. https://doi.org/10. 1080/14751798.2016.1199118

Banks AP, Gamblin DM, Hutchinson H (2020) Training fast and frugal heuristics in military decision making. Appl Cogn Psychol 34(3):699-709. https://doi.org/10.1002/acp.3658

Bonner HS (2018) The decision process: police officers' search for information in dispute encounters. Polic Soc 29(1):90-113. https://doi.org/10.1080/10439463.2016.1147040

Braun V, Clarke V (2006) Using thematic analysis in psychology. Qual Res Psychol 3(2):77-101. https://doi.org/10.1191/14780 88706qp063oa

Bryant D (2006) Rethinking ooda: toward a modern cognitive framework of command decision making. Mil Psychol 18(3):183206. https://doi.org/10.1207/s15327876mp1803_1

Burrell L, Noble A, Ridsdale L (2013) Decision-making by ambulance clinicians in London when managing patients with epilepsy: a qualitative study. Emerg Med J 30(3):236. https://doi. org/10.1136/emermed-2011-200388

Carsten O, Vanderhaegen F (2015) Situational awareness: vallid or fallacious? Cogn Technol Work 17:157-158. https://doi.org/10. 1007/s10111-015-0319-1

Catherwood D, Edgar GK, Sallis G, Medley A, Brookes D (2012) Fire alarm or false alarm?! Situation awareness and decisionmaking "bias" of firefighters in training exercises. Int J Emerg Serv 1(2):135-158. https://doi.org/10.1108/204708912112759 20

Cohen-Hatton SR, Butler PC, Honey RC (2015) An investigation of operational decision making in situ: incident command in the U.K. fire and rescue service. Hum Factors 57(5):793-804. https://doi. org/10.1177/0018720815578266

Cohen-Hatton SR, Honey RC (2015) Goal-oriented training affects decision-making processes in virtual and simulated fire and rescue environments. J Exp Psychol Appl 21(4):395-406. https://doi.org/ 10.1037/xap0000061

Comfort LK (2007) Crisis management in hindsight: cognition, communication, coordination and control. Public Adm Rev 67:189197. https://doi.org/10.1111/j.1540-6210.2007.00827.x

Correll J, Park B, Judd CM, Wittenbrink B (2007) Across the thin blue line: Police officers and racial bias in the decision to shoot. J Pers Soc Psychol 92(6):1006. https://doi.org/10.1037/0022-3514. 92.6.1006

Coskun E, Ozceylan D (2011) Complexity in emergency management and disaster response information systems (EMDRIS). Proceedings of the 8th International ISCRAM Conference, Lisbon, Portugal, May 2011. http://idl.iscram.org/files/coskun/2011/415_ Coskun+Ozceylan2011.pdf

Curnin S, Brooks B, Owen C (2020) A case study of disaster decisionmaking in the presence of anomalies and absence of recognition. J Contingencies Crisis Manag 28(2):110-121. https://doi.org/10. 1111/1468-5973.12290

DeSantis L, Ugarriza D (2000) The concept of theme as used in qualitative nursing research. West J Nurs Res 22:351-372

Dodd L, Moffat J, Smith J (2006) Discontinuity in decision-making when objectives conflict: a military command decision case study. 
J Oper Res Soc 57(6):643-654. https://doi.org/10.1057/palgrave. jors. 2602051

Endsley M (1995) Toward a theory of situational awareness in dynamic systems. Human Factors J 37(1):32-64. https://doi.org/10.1518/ 001872095779049543

Fielding N, Thomas H (2001) Qualitative interviewing. In: Gilbert N (ed) Researching social life, 3rd edn. Sage, pp 123-144

Flin R (1996) Sitting in the hot seat: leaders and teams for critical incident management. Wiley

Gamble KR, Vettel JM, Patton DJ, Eddy MD, Caroline Davis F, Garcia JO, Spangler DP, Thayer JF, Brooks JR (2018) Different profiles of decision making and physiology under varying levels of stress in trained military personnel. Int J Psychophysiol 131:73-80. https://doi.org/10.1016/j.ijpsycho.2018.03.017

Godwin H, Liversedge S, Kirkby J, Boardman M, Cornes K, Donnelly $\mathrm{N}$ (2015) The influence of experience upon information-sampling and decision-making behavior during risk assessment in military personnel. Vis Cogn 23(4):415-431. https://doi.org/10.1080/ 13506285.2015.1030488

Gunnarsson BM, WarrénStomberg M (2009) Factors influencing decision making among ambulance nurses in emergency care situations. Iny Emerg Nurs 17(2):83-89. https://doi.org/10.1016/j.ienj. 2008.10.004

Harman JL, Zhang D, Greening SG (2019) Basic processes in dynamic decision making: how experimental findings about risk, uncertainty, and emotion can contribute to police decision making. Front Psychol. https://doi.org/10.3389/fpsyg.2019.02140

Harris KR, Eccles DW, Freeman C, Ward P (2017) “Gun! Gun! Gun!”: an exploration of law enforcement officers' decision-making and coping under stress during actual events. Ergonomics 60(8):11121122. https://doi.org/10.1080/00140139.2016.1260165

Hemmatjo R, Zare S, Hajaghazadeh M, Allahyari T, Kazemi R (2019) Physiological strain and decision making affected by different cooling tactics following live-fire training. Human Factors Ergonomics Manuf 29(2):118-125. https://doi.org/10.1002/hfm.20763

Hine KA, Porter LE, Westera NJ, Alpert GP, Allen A (2019) What were they thinking? Factors influencing police recruits' decisions about force. Polic Soc 29(6):673-691. https://doi.org/10.1080/ 10439463.2018.1432612

House A, Power N, Alison LA (2013) A systematic review of the potential hurdles of interoperability to the emergency service in major incidents: Recommendations for solutions and alternatives. Cognitive Technical Work. https://doi.org/10.1007/ s10111-013-0259-6

Ishoy GA (2016) The theory of planned behavior and policing: how attitudes about behavior, subjective norms, and perceived behavioral control affect the discretionary enforcement decisions of police officers. Crim Justice Stud 29(4):345-362. https://doi.org/ 10.1080/1478601X.2016.1225362

Jederberg WW, Still KR, Briggs GB (2002) The utilization of risk assessments in tactical command decisions. Sci Total Environ 288(1-2):119-129. https://doi.org/10.1016/s0048-9697(01) 01118-4

Kahneman D (2011) Thinking, fast and slow. Farrar Straus and Giroux, New York

Kahneman D, Klein G (2009) Conditions for intuitive expertise: a failure to disagree. Am Psychol 64(6):515-526. https://doi.org/10. 1037/a0016755

Klein G, Calderwood R, Clinton-Cirocco A (2010) Rapid decision making on the fire ground: the original study plus a postscript. J Cogn Eng Decision Making 4(3):186-209. https://doi.org/10. 1518/155534310X12844000801203

Knighton RJ (2004) The psychology of risk and its role in military decision-making. Def Stud 4(3):309-334. https://doi.org/10.1080/ 1470243042000344786
Larsen RP (2001) Decision making by military students under severe stress. Mil Psychol 13(2):89-98. https://doi.org/10.1207/S1532 7876MP1302_02

Launder D, Perry C (2014) A study identifying factors influencing decision making in dynamic emergencies like urban fire and rescue settings. Int J Emerg Services 3(2):144-161. https://doi.org/ 10.1108/IJES-06-2013-0016

Leibold A, Lassen C, Lindenberg N, Graf B, Wiese C (2018) Is every life worth saving: does religion and religious beliefs influence paramedic's end-of-life decision-making? A prospective questionnaire-based investigation. Indian J Palliat Care 24(1):9-15. https:// doi.org/10.4103/IJPC.IJPC_128_17

Lima ME, de Araujo C (2018) The decision to shoot black suspects in Brazil: the police officer's dilemma. Race Soc Probl 10(2):101112. https://doi.org/10.1007/s12552-018-9225-5

Martínez-Fiestas M, Rodríguez-Garzón I, Delgado-Padial A (2020) Firefighter perception of risk: a multinational analysis. Saf Sci. https://doi.org/10.1016/j.ssci.2019.104545

Marusich LR, Bakdash JZ, Onal E, Yu MS, Schaffer J, O’ Donovan J, Höllerer T, Buchler N, Gonzalez C (2016) Effects of information availability on command-and-control decision making: performance, trust, and situation awareness. Hum Factors 58(2):301321. https://doi.org/10.1177/0018720815619515

McNeill P, Chapman S (2005) Research methods. Routledge, Oxon

Militello LG, Sushereba CE, Branlat M, Bean R, Finomore V (2015) Designing for military pararescue: naturalistic decision-making perspective, methods, and frameworks. J Occup Organ Psychol 88(2):251-272. https://doi.org/10.1111/joop.12114

Morgan D, Nica A (2020) Iterative thematic inquiry: a new method for analyzing qualitative data. Int J Qualitat Methods. https://doi.org/ $10.1177 / 1609406920955118$

Murdoch S (2019) Clinical decision-making and its place in paramedic practice. J Paramed Pract 11(5):1-5

Murphy-Jones G, Timmons S (2016) Paramedics' experiences of endof-life care decision making with regard to nursing home residents: an exploration of influential issues and factors. Emerg Med J 33(10):722-726. https://doi.org/10.1136/emermed-2015-205405

Nieuwenhuys A, Savelsbergh GJP, Oudejans RRD (2015) Persistence of threat-induced errors in police officers' shooting decisions. Appl Ergon 48:263-272. https://doi.org/10.1016/j.apergo.2014. 12.006

NIFC (2021) National interagency fire centre - incident management teams. https://www.nifc.gov/resources/firefighters/incident-manag ement-teams. Accessed July 2021

Nja O, Rake E (2009) A discussion of decision making applied in incident command. Int J Emergency Manage 6:55-72. https://doi. org/10.1504/ijem.2009.025173

O'Hara R, Johnson M, Hirst E et al (2015) Decision making and safety in ambulance service transitions. Emerg Med J 32(5):e2-e2. https://doi.org/10.1136/emermed-2015-204880.4

Okoli J, Watt J, Weller G, Wong WBL (2016) The role of expertise in dynamic risk assessment: A reflection of the problem-solving strategies used by experienced fireground commanders. Risk Manage 18(1):4-25. https://doi.org/10.1057/rm.2015.20

Oosterwold J, Sagel D, Berben S, Roodbol P, Broekhuis M (2018) Factors influencing the decision to convey or not to convey elderly people to the emergency department after emergency ambulance attendance: A systematic mixed studies review. BMJ Open 8(8):e021732. https://doi.org/10.1136/bmjopen-2018-021732

Penney G (2019) Exploring ISO31000 risk management during dynamic fire and emergency operations in Western Australia. Fire 2(2):21. https://doi.org/10.3390/fire2020021

Perona M, Rahman MA, O'Meara P (2019) Paramedic judgement, decision-making and cognitive processing: A review of the literature. Australasian J Paramed https://doi.org/10.33151/ajp.16.586 
Petticrew M, Roberts H (2006) Systematic reviews in the social sciences: a practical guide. Blackwell Publishing, Oxford

Pollock K (2013) Review of persistent lessons identified relating to interoperability from emergencies and major incidents since 1986. Emergency Planning College, Easingwold.

Prati G, Pietrantoni L, Saccinto E, Kehl D, Knuth D, Schmidt S (2013) Risk perception of different emergencies in a sample of European firefighters. Work 45:87-96. https://doi.org/10.3233/WOR-121543

Reay G, Rankin JA, Smith-MacDonald L, Lazarenko GC (2018) Creative adapting in a fluid environment: an explanatory model of paramedic decision making in the pre-hospital setting. BMC Emerg Med 18(1):42. https://doi.org/10.1186/s12873-018-0194-1

Ryan L, Halliwell D (2013) Paramedic decision-making-how is it done? J Paramed Pract 4(6):343-351

SAI Global (2013) SA/SNZ HB 436:2013 Risk management guidelines - Companion to AS/NZS ISO 31000. Standards Australia

Seiler S, Fischer A, Ooi YP (2010) An interactional dual-process model of moral decision making to guide military training. Mil Psychol 22(4):490-509. https://doi.org/10.1080/08995605.2010.513270

Shortland N, Alison L, Barrett-Pink C (2018) Military (in) decisionmaking process: A psychological framework to examine decision inertia in military operations. Theor Issues Ergon Sci 19(6):752772. https://doi.org/10.1080/1463922X.2018.1497726

Shortland N, Alison L, Thompson L (2020a) Military maximizers: Examining the effect of individual differences in maximization on military decision-making. Personality Individ Differ 163:110051. https://doi.org/10.1016/j.paid.2020.110051

Shortland N, Thompson L, Alison L (2020b) Police perfection: Examining the effect of trait maximization on police decision-making. Front Psychol 11:1817-1817. https://doi.org/10.3389/fpsyg.2020. 01817

Snowden D (2002) Complex acts of knowing: paradox and descriptive self awareness. J Knowl Manag 6(2):100-111. https://doi.org/10. $1108 / 13673270210424639$

Stanton NA, Salmon PM, Walker GH, Jenkins D (2009) Genotype and phenotype schemata as models of situation awareness in dynamic command and control teams. Int J Ind Ergon 39(3):480-489. https://doi.org/10.1016/j.ergon.2008.10.003

Thomas C (2020) Decision-making during Vuca crises: insights from the 2017 Northern California firestorm. J Bus Contin Emer Plan 14(1):82-94
Thompson MM, Hendriks T, Blais A-R (2018) Military ethical decision making: the effects of option choice and perspective taking on moral decision-making processes and intentions. Ethics Behav 28(7):578-596. https://doi.org/10.1080/10508422.2017.1372200

van den Heuvel C, Alison L, Power N (2014) Coping with uncertainty: police strategies for resilient decision-making and action implementation. Cogn Technol Work 16(1):25-45. https://doi.org/10. 1007/s10111-012-0241-8

Vanderhaegen F (2021) Heuristic-based method for conflict discovery of shared control between humans and autonomous systems - A driving automation case study.Robotics Auto Sys 146:103867

Verhage A, Noppe J, Feys Y, Ledegen E (2018) Force, stress, and decision-making within the Belgian police: the impact of stressful situations on police decision making. J Police Criminal Psychol 33(4):345-357. https://doi.org/10.1007/s11896-018-9262-4

Verhulst MJ, Rutkowski A (2018) Decision-making in the police work force: affordances explained in practice. Group Decis Negot 27(5):827-852. https://doi.org/10.1007/s10726-018-9587-5

Vickers JN, Lewinski W (2012) Performing under pressure: gaze control, decision making and shooting performance of elite and rookie police officers. Hum Mov Sci 31(1):101-117. https://doi.org/10. 1016/j.humov.20110

Wilkinson B, Cohen-Hatton S, Honey R (2021) Variation in exploration and exploitation in group decision making: Evidence from immersive simulations of major incident emergencies. J Contingencies Crisis Management. https://doi.org/10.1111/1468-5973. 12355

Wilson RS, Winter PL, Maguire LA, Ascher T (2011) Managing wildfire events: risk-based decision making among a group of federal fire managers managing wildfire events. Risk Anal 31(5):805818. https://doi.org/10.1111/j.1539-6924.2010.01534.x

Publisher's Note Springer Nature remains neutral with regard to jurisdictional claims in published maps and institutional affiliations. 